\title{
The Ste20 Family Kinases MAP4K4, MINK1, and TNIK Converge to Regulate Stress-Induced JNK Signaling in Neurons
}

\author{
() Martin Larhammar, ${ }^{1,3 *}$ Sarah Huntwork-Rodriguez, ${ }^{1,3 *}$ York Rudhard, ${ }^{2}$ Arundhati Sengupta-Ghosh, ${ }^{1}$ \\ and $\odot$ Joseph W. Lewcock ${ }^{1,3}$ \\ ${ }^{1}$ Department of Neuroscience, Genentech, Inc., San Francisco, California 94080, ${ }^{2}$ In Vitro Pharmacology, Evotec AG, Manfred Eigen Campus, 22419 \\ Hamburg, Germany, and ${ }^{3}$ Denali Therapeutics Inc., South San Francisco, California 94080
}

The c-Jun- $N$-terminal kinase (JNK) signaling pathway regulates nervous system development, axon regeneration, and neuronal degeneration after acute injury or in chronic neurodegenerative disease. Dual leucine zipper kinase (DLK) is required for stress-induced JNK signaling in neurons, yet the factors that initiate DLK/JNK pathway activity remain poorly defined. In the present study, we identify the Ste20 kinases MAP4K4, misshapen-like kinase 1 (MINK1 or MAP4K6) and TNIK Traf2- and Nck-interacting kinase (TNIK or MAP4K7), as upstream regulators of DLK/JNK signaling in neurons. Using a trophic factor withdrawal-based model of neurodegeneration in both male and female embryonic mouse dorsal root ganglion neurons, we show that MAP4K4, MINK1, and TNIK act redundantly to regulate DLK activation and downstream JNK-dependent phosphorylation of c-Jun in response to stress. Targeting MAP4K4, MINK1, and TNIK, but not any of these kinases individually, is sufficient to protect neurons potently from degeneration. Pharmacological inhibition of MAP4Ks blocks stabilization and phosphorylation of DLK within axons and subsequent retrograde translocation of the JNK signaling complex to the nucleus. These results position MAP4Ks as important regulators of the DLK/JNK signaling pathway.

Key words: DLK; JNK; MAP4K4; MINK1; neurodegeneration; TNIK

Significance Statement

Neuronal degeneration occurs in disparate circumstances: during development to refine neuronal connections, after injury to clear damaged neurons, or pathologically during disease. The dual leucine zipper kinase (DLK)/c-Jun- $N$-terminal kinase (JNK) pathway represents a conserved regulator of neuronal injury signaling that drives both neurodegeneration and axon regeneration, yet little is known about the factors that initiate DLK activity. Here, we uncover a novel role for a subfamily of MAP4 kinases consisting of MAP4K4, Traf2- and Nck-interacting kinase (TNIK or MAP4K7), and misshapen-like kinase 1 (MINK1 or MAP4K6) in regulating DLK/JNK signaling in neurons. Inhibition of these MAP4Ks blocks stress-induced retrograde JNK signaling and protects from neurodegeneration, suggesting that these kinases may represent attractive therapeutic targets.

\section{Introduction}

Postmitotic mouse neurons express all three of the c-Jun- $N$ terminal kinase (JNK) genes present in vertebrates (JNK1-3 or MAPK8-10), which have diverse roles that range from regulation of axonal growth and synaptic stability to directing apoptosis

Received April 3, 2017; revised Sept. 13, 2017; accepted 0ct. 2, 2017.

Author contributions: M.L., S.H.-R., and J.W.L. designed research; M.L., S.H.-R., Y.R., and A.S.-G. performed research; M.L., S.H.-R., Y.R., A.S.-G., and J.W.L. analyzed data; M.L., S.H.-R., and J.W.L. wrote the paper.

We thank Jeffrey Eastham-Anderson, Hai Ngu, and Oded Foreman for assistance with image quantification; Weilan Ye and Philip Vitorino for providing Map4k4 conditional knock-out mice and helpful discussions; and Chudi Ndubaku for providing MAP4K4 inhibitors and associated information for their use.

M.L., S.H-R., A.S-G., and J.W.L. are current or former employees of Genentech, Inc. Y.R. declares no competing financial interests.

${ }^{*}$ M.L. and S.H.-R. contributed equally to this work. after insult. JNK1 displays high activity under physiological conditions, whereas JNK2 and JNK3 display low basal levels of activity that are induced by cellular stress (Coffey et al., 2002). Consistent with this, genetic deletion of JNK1 does not affect neuronal injury signaling, whereas animals lacking expression of JNK2 and/or JNK3 display an attenuated response to a broad range of insults (Hunot et al., 2004; Ries et al., 2008; Fernandes et al., 2012; Genabai et al., 2015). In the peripheral nervous system, JNK2/3-dependent phosphorylation of the transcription factor c-Jun is required for axon regeneration after injury (Barnat et al.,

Correspondence should be addressed to Joseph W. Lewcock, Ph.D., Denali Therapeutics Inc., 151 Oyster Point Blvd, South San Francisco, CA 94080. E-mail: lewcock@dnli.com.

DOI:10.1523/JNEUROSCI.0905-17.2017

Copyright $\odot 2017$ the authors $\quad 0270-6474 / 17 / 3711074-11 \$ 15.00 / 0$ 
2010; Nix et al., 2011). Conversely, the same signaling events result in axon degeneration and apoptosis during neuronal development, after acute injury in the CNS, and in models of chronic neurodegenerative disease. These findings have generated significant interest in therapeutic approaches to modulate JNK activity either directly or indirectly.

Stress-induced JNK signaling in neurons requires the upstream mixed-lineage kinase dual leucine zipper kinase (DLK or MAP3K12) (Hirai et al., 2005; Miller et al., 2009; Ghosh et al., 2011; Pozniak et al., 2013). After neuronal injury, DLK activation induces retrograde propagation of downstream stress signals, including phosphorylated JNK (p-JNK), to the nucleus, resulting in c-Jun phosphorylation (Cavalli et al., 2005; Lindwall and Kanje, 2005; Ghosh et al., 2011; Fernandes et al., 2012; Shin et al., 2012; Huntwork-Rodriguez et al., 2013; Simon et al., 2016; Larhammar et al., 2017). This translocation event requires the dynein-associated scaffolding protein JIP3, suggesting that translocation of p-JNK occurs via dynein-dependent retrograde transport (Cavalli et al., 2005). Genetic deletion or pharmacological inhibition of DLK is sufficient to abrogate stress-induced gene expression changes broadly and results in potent protection from neurodegeneration in many experimental settings and inhibition of axon regeneration in others (Miller et al., 2009; Ghosh et al., 2011; Shin et al., 2012; Watkins et al., 2013; Larhammar et al., 2017; Le Pichon et al., 2017).

Recent work has shed light on the mechanisms of DLK/JNK pathway activation (Collins et al., 2006; Huntwork-Rodriguez et al., 2013; Valakh et al., 2013; Wu et al., 2015; Simon et al., 2016), but the factors directly upstream of DLK remain poorly defined. Previous studies have demonstrated that certain MAP4 kinases are capable of activating JNK in heterologous systems, with some data suggesting that MAP4K4 may also modulate JNK signaling in motor neurons (Machida et al., 2004; Yang et al., 2013b). In the present study, we perform a high-content imaging screen using the nerve growth factor (NGF) withdrawal model of developmental neurodegeneration in embryonic dorsal root ganglion (DRG) neurons to demonstrate that the germinal center kinase-IV (GCK-IV) subfamily of Ste20 kinases MAP4K4, misshapen-like kinase 1 (MINK1 or MAP4K6), and Traf2- and Nck-interacting kinase (TNIK or MAP4K7) act redundantly to regulate retrograde DLK/ JNK signaling in neurons.

\section{Materials and Methods}

Animal models. All experiments were performed under animal protocols approved by the Animal Care and Use Committee at Genentech. CD-1 embryonic day 12.5 (E12.5) to E13.5 mouse embryos of either sex were used for DRG preparations. Rat E14.5 embryos were used for the smallmolecule screen. MAP4K $4^{\text {loxp }}$ animals were generated as described previously (Vitorino et al., 2015). MAP4K $4^{\text {loxp }}$ animals were crossed to a transgenic pCAGG-Cre/ERT2 line, which contains an insertion of a construct encoding a Cre-estrogen receptor fusion protein behind a CAGGS promoter to give ubiquitous expression of the Cre fusion protein (Jackson Laboratories) (Pozniak et al., 2013; Watkins et al., 2013).

Small-molecule inhibitors. GNE-495, MAP4Ki_29, MAP4Ki_10, MAP4Ki_26, and DLK inhibitor GNE-3511 were generated as described previously (Crawford et al., 2014; Ndubaku et al., 2015; Patel et al., 2015; Vitorino et al., 2015). Cycloheximide was purchased from Sigma-Aldrich (catalog \#C7698).

Antibodies. The following antibodies were used: rabbit anti-DLK monoclonal clone 49-5 (1:1000 for Western blot and staining, produced at Genentech according to Hirai et al., 2002); rabbit anti-DLK (1:1000; Genetex, catalog \#GTX124127); rabbit anti-DLK phospho-T43 (HuntworkRodriguez et al., 2013), anti-p-JNK (1:500; Cell Signaling Technology, catalog \#9251); anti-p-c-Jun (1:250 for Western blot and 1:500 for staining; Cell Signaling Technology, catalog \#9261); anti- $\beta$ III-tubulin (Tuj-1,
1:1000, Covance, catalog \#MMS-435P-250 and Tuj1-488, BioLegend, catalog \#801203), anti-Mink1 (1:500; Novus, catalog \#NBP1-22989), anti-TNIK (1:500; Genetex, catalog \#GTX13141), anti-MAP4K4 (1:500; Cell Signaling Technology, catalog \#3485), anti-cleaved-caspase-3 (1: 400; Cell Signaling Technology, catalog \#9661), mouse anti- $\beta$-actin (1: 15,000; Abcam, catalog \#ab6276), and anti-GAPDH (1:1000; Genetex, catalog \#GTX627408). DLK staining of DRGs was done using an antiDLK monoclonal antibody with a human backbone at a 1:1000 dilution. To produce this antibody, rabbits were immunized with a C-terminal portion of DLK as described previously (Hirai et al., 2002). Monoclonal antibodies were generated from these rabbits and the backbone of one positive clone (49-5) was humanized to allow for costaining with other antibodies with rabbit backbones.

Primary neuronal culture. DRGs were dissected from E12.5 to E13.5 mouse embryos, trypsinized (except in the case of explants), and cultured in F12 medium containing N3 supplement, $40 \mathrm{~mm}$ glucose, and $25 \mathrm{ng} / \mathrm{ml}$ NGF. Primary DRG neurons were plated in poly-D-lysine and laminincoated Corning chamber slides (BioCoat; BD Biosciences) or Corning 24 -well plates. The day after plating, $3 \mu \mathrm{M}$ Cytosine $\beta$-Darabinofuranoside (AraC; Sigma-Aldrich) or $1 \mu \mathrm{M}$ 5-fluorouracil and 1 $\mu \mathrm{M}$ uridine (Sigma-Aldrich) was added to the medium to inhibit mitosis. NGF withdrawal was conducted at 3-5 d in vitro by replacing cell medium with medium containing no NGF and $50 \mu \mathrm{g} / \mathrm{ml}$ antiNGF antibody (Genentech) or $15 \mu \mathrm{g} / \mathrm{ml}$ anti-NGF antibody (AB1528SP; Millipore).

For neurodegeneration and histological analysis, cells were fixed in $4 \%$ PFA. For molecular analysis, cells were lysed in radioimmunoprecipitation assay (RIPA) (see details below). In experiments using cultured primary DRG neurons from the MAP4K $4^{\text {loxp/loxp }}$; Cre-ERT2 mouse line, recombination of the floxed sites in MAP4K4 was induced by addition of $1 \mu \mathrm{M} 4$-hydroxytamoxifen (Sigma-Aldrich) to the medium for $24 \mathrm{~h}$.

For siRNA experiments, dissociated DRGs were transfected using the Amaxa Nucleofection System P3 Primary Cell Kit. Tnik siRNA (sense 5' -GCT CAA GAC TCA ACC GAC ATT-3' , antisense 5' -TGT CGG TTG AGT CTT GAG CTT-3') was synthesized at Genentech. Mink1 siRNA was obtained from Life Technologies (catalog \#s78611). Control siRNA was obtained from Dharmacon (ON-TARGETplus Nontargeting siRNA \#1, catalog \#D-001810-01-05).

Immunocytochemistry. DRG neurons plated on 8-well slides were fixed for $15-30 \mathrm{~min}$ in $4 \%$ paraformaldehyde, blocked in PBS containing 5\% BSA and $0.3 \%$ Triton X-100 and incubated overnight with primary antibody diluted in blocking buffer. The slides were washed in PBS and secondary antibodies (goat anti-rabbit Alexa Fluor 488, goat anti-human Alexa Fluor 568, and goat anti-mouse Alexa Fluor 647 Life Technologies) were added in blocking buffer for $1 \mathrm{~h}$ at room temperature. The slides were washed in PBS and mounted in Fluoromount-G containing DAPI (Southern Biotech).

Images of DRG cultures were acquired using a fluorescent microscope (DM5500; Leica) and Advanced Fluorescence Application Suite software with a DFC360 camera using Leica $20 \times / 0.70$ or Leica $40 \times / 0.75$ numerical aperture objectives. All images were acquired at room temperature. Images of Campenot chambers were acquired under a confocal microscope (LSM710 with a LSM-TPMT camera, Carl Zeiss) with Zen 2010 software using a Zeiss $20 \times / 0.8$ numerical aperture objective and singleplane imaging.

Images shown were processed in Adobe Photoshop to enhance visibility by adjusting brightness and contrast. Within an individual figure, all images were subjected to the same postacquisition processing.

High-content p-c-Jun imaging and quantification assay. Quantitative characterization of MAP4K inhibitors with $\mathrm{p}$-c-Jun as a readout was performed as described previously (Rudhard et al., 2015). In brief, E14.5 dorsal root ganglia from rats were dissected, dissociated, and plated on a bed of astrocytes. After $4 \mathrm{~d}$, NGF was withdrawn and anti-NGF antibody was added together with inhibitors. After $2 \mathrm{~h}$, the cells were fixed and stained with anti-p-c-Jun, DRAQ5, and HuD antibodies. Images were acquired with an Opera High Content Screening System using a $10 \times$ lens and laser lines 488, 532, and $635 \mathrm{~nm}$. Six images were taken per well and evaluated using Acapella script-based algorithms to detect nuclei and axon area. Detected nuclei were used to identify and count cells and a 
minimum nuclear area threshold criterion was applied to select for live cells. The nuclear stencil was also used to quantify the mean intensities of $\mathrm{p}-\mathrm{c}$-Jun and $\mathrm{HuD}$, respectively. Intensity threshold criteria were set for $\mathrm{p}$-c-Jun and $\mathrm{HuD}$ to flag positive cells and to determine the proportion of neurons ( $\mathrm{HuD}$ threshold-positive cells) labeled for $\mathrm{p}$-c-Jun ( $\mathrm{p}$-c-Jun threshold-positive cells). Pharmacological inhibition of $\mathrm{p}$-c-Jun was determined with minimal p-c-Jun levels in NGF controls (full inhibition, $0 \%$ ) and maximal in anti-NGF controls (no inhibition, 100\%). Concentration-response curves were analyzed with Excel Fit software using the 4 Parameter Logistic Model or Sigmoidal Dose-Response Model.

Western blot. DRG cultures were lysed in RIPA buffer containing complete protease inhibitor mixture and PhosSTOP phosphatase inhibitor mixture (Roche). Isolated axons were lysed directly in Western blot loading buffer. Samples were loaded on NuPAGE 4-12\% Bis-Tris gels (Invitrogen) and subjected to standard immunoblotting procedures. Blots were visualized with chemiluminescence on a Chemidoc (Bio-Rad) or with fluorescence using an Odyssey detection system (LI-COR). Quantification of Western blots were conducted using Image lab (version 5.2.1, 2015; Bio-Rad) or Image Studio Lite (version 5.2.5, 2014; LI-COR) software. Relative protein levels were determined by normalization to loading controls.

Kinase activity. The half-maximal inhibitory concentration $\left(\mathrm{IC}_{50}\right)$ for GNE-495 of TNIK activity was determined using a TNIK kinase assay (V4158; Promega). In brief, GNE-495 at 12 concentrations between 6 pM and $10 \mu \mathrm{M}$ was incubated with $30 \mathrm{ng}$ of recombinant human TNIK protein (aa 1-367) in Promega kinase reaction buffer in a white 96-well plate for $60 \mathrm{~min}$ according to the manufacturer's protocol. ADP-Glo luminescent kinase detection reagent (V9101) were added to the wells and incubated for another $30 \mathrm{~min}$. Luminescence was recording using a Synergy H1 Hybrid Multi-Mode Reader equipped with Software Gen5 (version 2.09; BioTek Instruments). Measurements were performed on triplicate samples (integration time $0.5 \mathrm{~s}$ ) and average values were used to determine $\mathrm{IC}_{50}$ for GNE-495. IC 50 for MAP4Ki_29, MAP4Ki_10, MAP4Ki_26, MAPK4, and MINK1 was determined at Invitrogen according to standard protocols.

Cycloheximide and lambda-phosphatase treatment of DRGs. Dissociated E13.5 DRG neurons were incubated with a final concentration of $5 \mu \mathrm{M}$ cycloheximide (Sigma-Aldrich) in either NGF-containing medium (control) or anti-NGF medium ( $15 \mu \mathrm{g} / \mathrm{ml}$ sheep anti-NGF) and treated with 800 nM GNE-495 or DMSO. Cells were lysed after 4 or $8 \mathrm{~h}$ and subjected to Western blot for DLK. Anti-NGF samples (DMSO and GNE-495) were normalized to loading control and + NGF DMSO controls for each time point and relative protein levels were used for statistical analysis.

Lambda protein phosphatase treatment was performed as described previously (Huntwork-Rodriguez et al., 2013). In brief, primary DRG lysates were incubated with $800 \mathrm{U}$ lambda phosphatase (New England Biolabs, catalog \#P0753S) for $45 \mathrm{~min}$ at $30^{\circ} \mathrm{C}$ in RIPA buffer supplemented with a final concentration of $1 \times \mathrm{PMP}$ buffer and $1 \mathrm{mM} \mathrm{MnCl} \mathrm{m}_{2}$ according to the manufacturer's protocol (20 $\mu$ l reaction volume).

Campenot chamber and axon isolation experiments. Compartmentalized (Campenot) chamber assays were performed essentially as described previously (Campenot, 1977; Nikolaev et al., 2009). In brief, Campenot chambers were assembled using poly-D-lysine and laminin-coated $35 \mathrm{~mm}$ tissue culture dishes scratched with a pin rake (Tyler Research) to generate tracks for axonal growth and containing a Teflon divider (Camp8; Tyler Research) to create a central cell body chamber flanked by two axon chambers. Dissociated E12.5 DRGs were plated in the cell body compartment in Neurobasal methylcellulose medium supplemented with L-glutamine, glucose, B27, and penicillin-streptomycin (Invitrogen) with NGF. The following day, the axonal compartments were filled with culture medium containing $4 \mathrm{mg} / \mathrm{ml}$ methylcellulose and $50 \mathrm{ng} / \mathrm{ml}$ NGF. NGF was withdrawn from axonal compartments after 3-5 d in vitro. MAP4K inhibitor was added to the axonal compartment at the time of NGF withdrawal. Cells were fixed in $4 \%$ PFA in 3\% sucrose after $6 \mathrm{~h}$.

Isolation of DRG axons was performed essentially as described previously (Unsain et al., 2014). In brief, 30 E12.5-E13.5 DRGs were plated as explants on PDL, laminin, and collagen-coated porous cell culture filters
(Falcon, catalog \#87717), treated with AraC for $24 \mathrm{~h}$, and grown for $3 \mathrm{~d}$ in medium containing $50 \mu \mathrm{g} / \mathrm{ml} \mathrm{NGF}$ in the bottom (axonal) compartment and $25 \mu \mathrm{g} / \mathrm{ml} \mathrm{NGF}$ in the upper (explant) compartment (see illustration in Fig. 5A). NGF was withdrawn in the presence of inhibitor GNE-495 or equal volumes of DMSO for 1, 3, and $6 \mathrm{~h}$. Axons and explants were separated and subjected to Western blot analysis. The activated fraction of DLK was calculated based on the Western blot banding pattern; shifted high molecular weight $\left(\mathrm{DLK}_{\text {shiff }}\right)$ was divided by total DLK protein $\left(\mathrm{DLK}_{\text {total }}\right)$ and normalized to actin signal using Image Studio Lite (version 5.2.5, 2014; LI-COR; see Fig. 5E).

Image quantification. Axon degeneration of DRG explants was quantified blindly on a scale of $0-5$ as described previously (Ghosh et al., 2011), in which 0 equals no degeneration and 5 equals complete degeneration. Representative images were used to define intermediate stages of degeneration. Axon degeneration in dissociated DRG neurons was quantification using MetaMorph software (Molecular Devices). The number of "connected sets" was used to measures axon fragmentation (a completely undegenerated culture would measure as one connected set and a greater number means more fragmented axons).

Quantification of cleaved Caspase-3 in primary DRGs was conducted as described previously (Ghosh et al., 2011; Larhammar et al., 2017).

Quantification of DLK and p-JNK intensity was performed in ImageJ 64-bit version 1.84v. Regions of Interest (ROIs) were drawn around 10 cell bodies per image using the tubulin channel. These ROIs were then used as overlays for the DLK and p-JNK images. For each cell ROI, the average intensity within that cell was measured. Four images per condition were quantified for a total of 40 cells. To measure the relative intensity of DLK and p-JNK compared with the + NGF control, each of the 40 measurements was divided by the mean intensity of the +NGF control.

Quantification of the number of p-c-Jun-positive cells in Campenot chambers was performed in ImageJ 64-bit version 1.84v. RGB images were converted to 16-bit grayscale, the Threshold function was used to highlight pixel intensities $>13$, and the image was converted to binary and inverted. The number of p-c-Jun-positive cells was quantified using the "Analyze Particles" function.

Statistical analysis. Statistical analyses were performed using GraphPad Prism version 6.0). Comparisons between two groups were analyzed by Student's two-sample $t$ test and comparisons between multiple groups were analyzed using one-way ANOVA followed by Bonferroni's or Dunnett's multiple-comparisons test. Information about sample sizes and the specific statistical test used for each experiment is provided in the figure legends. Further details on the data analysis are provided in this section for individual methods. Data are presented as mean \pm the SEM unless stated otherwise. Statistical significance is illustrated in the figures as ${ }^{*} p<0.05,{ }^{* *} p<0.01,{ }^{* * *} p<0.001$, and ${ }^{* * * *} p<0.0001$.

\section{Results}

To identify factors that regulate DLK/JNK stress signaling, we used the NGF withdrawal model in primary embryonic DRG neurons, which mimics the competition for NGF by TrkA-expressing DRG neurons during development (Oppenheim, 1991). Many of the signaling mechanisms identified in this model display a conserved function in other contexts, making it an attractive model for the identification of factors that regulate neurodegeneration (Simon et al., 2012; Watkins et al., 2013; Yang et al., 2013a; Rudhard et al., 2015; Simon et al., 2016; Larhammar et al., 2017). Upon NGF deprivation in DRG neurons, phosphorylation of $\mathrm{c}$-Jun (p-c-Jun) is rapidly induced in a DLK/JNK dependent fashion and thus provides a robust readout of pathway activation (Ghosh et al., 2011; Huntwork-Rodriguez et al., 2013). We modified our previously described high-content platform (Rudhard et al., 2015) to monitor nuclear p-c-Jun in neurons after $2 \mathrm{~h}$ of NGF withdrawal and screened a library of compounds with diverse activities for their ability to suppress the appearance $\mathrm{p}-\mathrm{c}$ Jun in DRGs.

This screen led to the identification of a set of previously characterized selective inhibitors of MAP4K4 (GNE-495, MAP4Ki_10, 
A + NGF anti-NGF
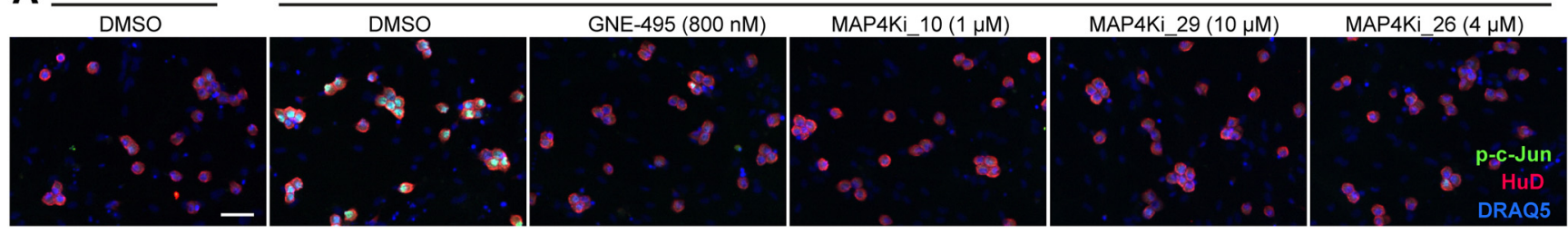

\section{B $\% \mathrm{p}$-cJun neurons number of nuclei}
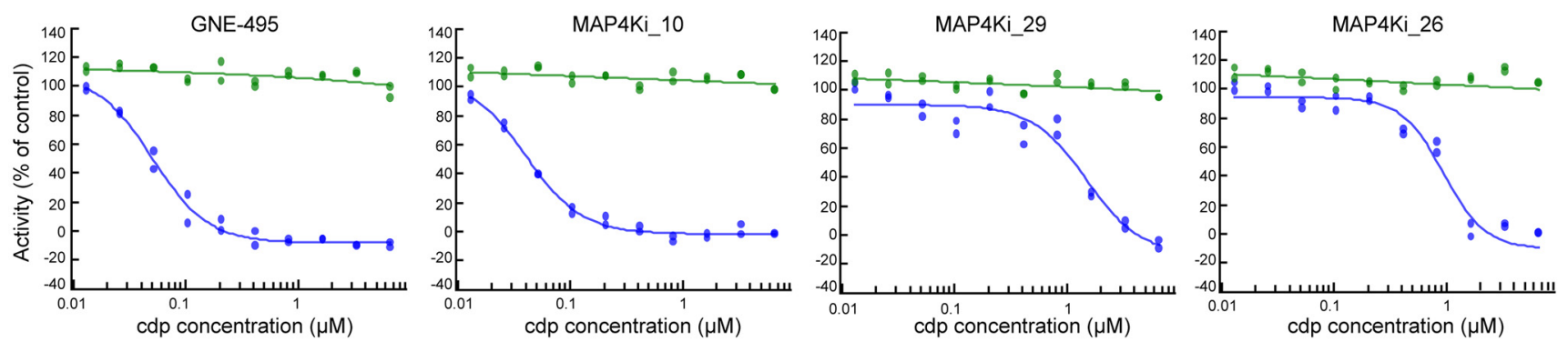

Figure 1. Small-molecule screen in primary DRG neurons identifies MAP4K4 inhibitors as regulators of JNK signaling. $\boldsymbol{A}$, Representative immunofluorescence images showing c-Jun phosphorylation in embryonic DRG cultures after NGF withdrawal (2 h). Small-molecule inhibitors targeting MAP4K4 block p-c-Jun upregulation. Green: p-c-Jun; blue: DRAQ5 (nuclei); red: HuD (cytoplasm). Scale bar, $50 \mu \mathrm{m}$. IC ${ }_{50}(\mathrm{~nm})$ : GNE-495, 2.24; MAP4Ki_10, 1.05; MAP4Ki_29, 14.5; and MAP4Ki_26, 16.0.Concentrations of inhibitors: GNE-495, 200 nm; MAP4Ki_10,833 nm; MAP4Ki_29, 6.67 $\mu$ m; and MAP4Ki_26,3.30 $\mu \mathrm{m}$. DMSO was used as a control. B, Dose-response curves showing the ratio of p-c-Jun-positive nuclei after $2 \mathrm{~h}$ NGF withdrawal treated with varying concentrations of four different MAP4K4 inhibitors to that in cultures treated with vehicle alone (expressed as a percentage). Each concentration was tested in duplicate wells, with both values plotted. $\mathrm{EC}_{50} \mathrm{COncentrations}$ $(\mu \mathrm{M})$ calculated from the mean IC ${ }_{50}$ values of two plate replicates are as follows: GNE-495, 0.0476; MAP4Ki_10,0.0343; MAP4Ki_29, 1.605; and MAP4Ki_26, 1.027.

MAP4Ki_29, MAP4Ki_26 (Crawford et al., 2014; Ndubaku et al., 2015; Vitorino et al., 2015)) that all blocked p-c-Jun induction with minimal toxicity as measured by quantification of neuronal nuclei (Fig. $1 A, B$ ). The $\mathrm{IC}_{50}$ of p-c-Jun inhibition for these compounds were consistent with the previously reported cellular $\mathrm{IC}_{50}$ values for MAP4K4 (Crawford et al., 2014; Ndubaku et al., 2015; Vitorino et al., 2015), suggesting that this effect was a result of on-target kinase inhibition rather than a nonselective off-target activity.

To validate the activity of the MAP4K4 inhibitors identified in our screen, we first performed immunoblotting of primary DRG neuron lysates after NGF deprivation. Consistent with our imaging data, all four of the MAP4K4 inhibitors tested were able to reduce levels of p-c-Jun potently, as shown by Western blot (Fig. $2 A$ ). Furthermore, these MAP4K4 inhibitors also repressed activation of the MAP3 kinase DLK, as measured by a suppression of phosphorylation-dependent increase in DLK protein abundance and molecular weight that occurs after NGF withdrawal (HuntworkRodriguez et al., 2013) (Fig. 2A). The effect of GNE-495 treatment on DLK abundance and molecular weight was similar to that observed after addition of lambda phosphatase to lysates (Fig. $2 B)$, providing additional support for our conclusion that MAP4K activity is required for DLK phosphorylation and activation (Fig. 2B). As expected based on the levels of p-c-Jun, examination of p-JNK in the same experiment revealed that GNE-495 treatment was also sufficient to attenuate the NGF-withdrawalinduced increase in JNK signaling downstream of DLK.

We next investigated whether MAP4K inhibitor treatment affects phosphorylation of DLK specifically at residue threonine 43 (T43), a site required for DLK stabilization that is known to exhibit phosphorylation in stressed neurons (HuntworkRodriguez et al., 2013). Immunoblotting with a $\mathrm{DLK}^{\mathrm{T} 43}$-specific antibody (Huntwork-Rodriguez et al., 2013) demonstrated that GNE-495 treatment efficiently blocked the T43 phosphorylation that occurs after NGF withdrawal (Fig. 2C). Based on this result, we sought to determine whether inhibition of MAP4K4 also af- fects DLK protein stability. To do this, NGF-deprived DRG cultures were treated with the protein synthesis inhibitor cycloheximide in the presence of absence of GNE-495 for either 4 or $8 \mathrm{~h}$ (Fig. 2D). Treatment of neurons with GNE-495 resulted in significantly lower levels of DLK protein at both time points, indicating reduced DLK stability upon MAP4K4 inhibition (Fig. $2 D, E)$. Together, these results demonstrate that treatment with inhibitors targeting MAP4K4 is sufficient to attenuate the phosphorylation and stabilization of DLK that occurs after NGF withdrawal. These results, together with previously published data showing that the phosphorylation of JNK and c-Jun after NGF deprivation is DLK dependent (Ghosh et al., 2011), suggest that inhibition of MAP4K4 blocks JNK pathway activation through a DLK-dependent mechanism.

To determine whether treatment with MAP4K4 inhibitors was sufficient to attenuate axonal degeneration and neuronal apoptosis in DRG neurons, we treated DRG explants with four different MAP4K4 inhibitors (GNE-495, MAP4Ki_10, MAP4Ki_29, and MAP4Ki_26) at the minimal concentration required to achieve full suppression of c-Jun phosphorylation. Examination of DRG explants after $20 \mathrm{~h}$ of NGF withdrawal revealed that all compounds were able to elicit strong protection from axon degeneration (Fig. $3 A, B)$. To test whether the effect of MAP4K4 inhibition similarly blocked neuronal apoptosis, the number of cells immunopositive for activated Caspase-3 was quantified. Treatment with MAP4K4 inhibitor GNE-495 reduced the number of DRG cell bodies labeled for activated Caspase- $38 \mathrm{~h}$ after NGF withdrawal to a similar extent as treatment with the neuroprotective DLK inhibitor GNE-3511 (Patel et al., 2015) (Fig. 3C,D). Therefore, MAP4K4 inhibitors are neuroprotective in the NGF withdrawal model.

Broad profiling of GNE-495 against 244 kinases using a panel of in vitro enzymatic assays demonstrated that this molecule is a highly selective inhibitor (Crawford et al., 2014; Ndubaku et al., 2015). However, inhibition of additional kinases may contribute to the effects observed. For instance, the kinase domain of MAP4K4 shares $100 \%$ sequence identity with two other members 
A

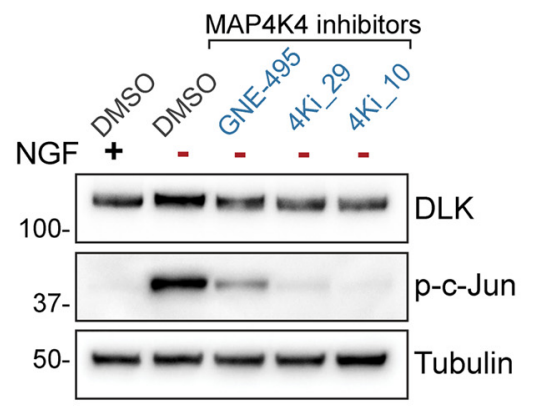

D

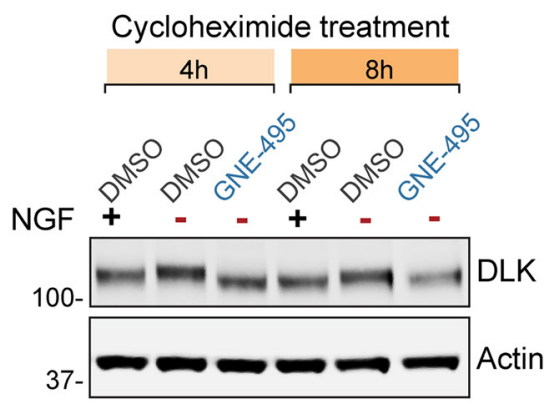

B

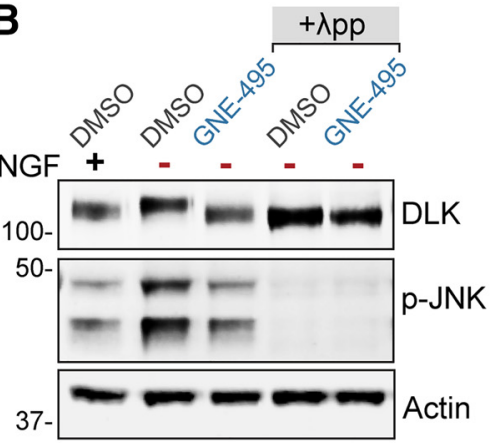

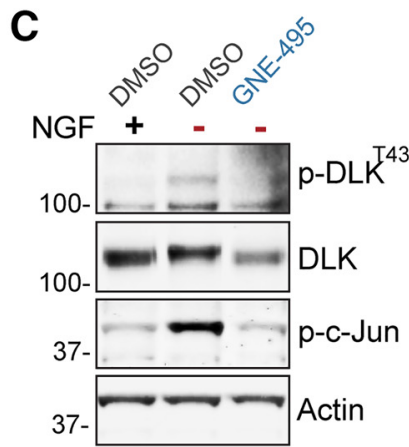

E

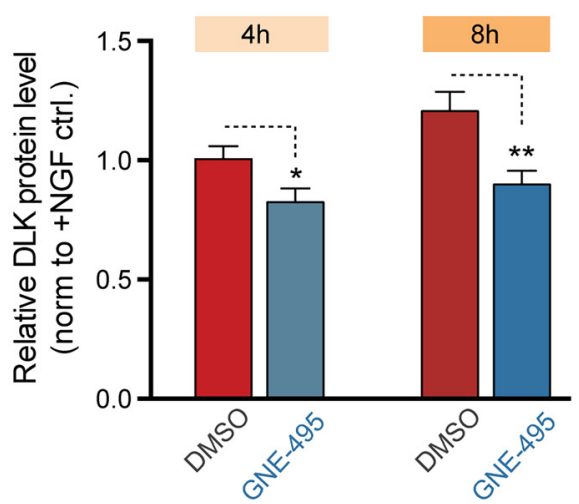

Figure 2. Inhibitors targeting MAP4K4 reduce DLK/JNK pathway activation after NGF deprivation. $\boldsymbol{A}$, Western blot analysis of NGF-deprived DRG neuron lysates demonstrate phosphorylation of c-Jun and activation of DLK as measured by increased DLK abundance and a shift in molecular weight after $3 \mathrm{~h}$. Treatment with inhibitors targeting MAP4K4, GNE-495 (800 nM), MAP4Ki_ 10 (1 $\mu \mathrm{M}$ ), and MAP4Ki_29 $(10 \mu \mathrm{M})$, block p-c-Jun and DLK activation. B, Treatment with GNE-495 (800 nM) blocks phosphorylation of JNK (p-JNK) and DLK (shift in molecular weight) after NGF deprivation. Lambda protein phosphatase $(\lambda \mathrm{pp})$ treatment of DRG samples confirms that the increased molecular weight of DLK is due to phosphorylation. C, Phosphorylation of DLK on T43 detected by an antibody targeting phosphorylated DLK-T43 (p-DLK ${ }^{\top 43}$ ) is induced by NGF withdrawal. Treatment with GNE-495 treatment suppresses p-DLK ${ }^{\top 43}$ and downstream p-c-Jun. D, Analysis of DLK protein stability after 4 and $8 \mathrm{~h}$ of NGF withdrawal using the protein synthesis inhibitor cycloheximide $(5 \mu \mathrm{M})$. E, GNE-495 treatment attenuates the increase in DLK protein stability in response to stress, as demonstrated by NGF deprivation 4 and $8 \mathrm{~h}$ in the presence of cycloheximide. Relative DLK protein levels were normalized to cycloheximide-treated DRG control lysates in the presence of NGF for each time point. DLK $4 \mathrm{~h}: p=0.03,8 \mathrm{~h}: p=0.004 . n=8-10 /$ condition from six independent experiments. Student's $t$ test was used for statistical analysis. Data are represented as mean \pm SEM, ${ }^{*} p<$ $0.05,{ }^{* *} p<0.01$.

of the GCK-IV kinase subfamily of the Ste20 family of kinases, TNIK and MINK1, that were not present in this kinase panel (Dan et al., 2001; Crawford et al., 2014). Therefore, it is possible that GNE-495 and related compounds targeting MAP4K4 also inhibit TNIK and MINK1, which could contribute to the neuroprotection observed. To explore this possibility, we determined the $\mathrm{IC}_{50}$ of each of the four MAP4K4 inhibitors in a cell-free biochemical kinase activity assay with purified MINK1 (GNE495: 5.2 nM, MAP4Ki_10: $2.5 \mathrm{nM}$, MAP4Ki_29: $40.3 \mathrm{nM}$, and MAP4Ki_26: $27.5 \mathrm{nM}$ ). The $\mathrm{IC}_{50}$ of GNE-495 was also tested for TNIK1 using purified recombinant human protein and determined to be $4.8 \mathrm{nM}$. Comparison of these values with the previously reported biochemical potency of these compounds for MAP4K4 (Crawford et al., 2014; Ndubaku et al., 2015; Vitorino et al., 2015) revealed only an $\sim 2-3 \times$ difference in potency between MAP4K4 and TNIK/MINK1.

Based on these results, we sought to validate genetically whether loss of MAP4K4 alone is sufficient to attenuate stress-induced DLK/ JNK signaling in neurons. Map4k4 deletion was induced in primary DRG neurons using mice carrying a floxed allele of Map4k4 (Vitorino et al., 2015) and a tamoxifen-inducible Cre (Cre-ERT2) driven by the CAG promoter (Map4k4 $\left.4^{\text {loxp/loxp }} ; \mathrm{Cre}^{+}\right)$. This strategy circumvented the early embryonic lethality of Map $4 k 4$ knock-out mice (Xue et al., 2001), as well as the confounding effects of incomplete excision that arise using neuron-specific Cre lines (Simon et al., 2016). To induce recombination of the Map4k4 allele, cultured embryonic DRG neurons from Map4k4 $4^{\text {loxp/loxp; }}$ $\mathrm{Cre}^{+}$mice were treated with 4-hydroxytamoxifen (4-OHT) for $24 \mathrm{~h}$. This treatment resulted in loss of MAP4K4 expression, but did not affect the induction of DLK and p-c-Jun after NGF withdrawal (Fig. 4A). To determine whether MINK1 or TNIK is responsible for JNK pathway activation upon NGF deprivation rather than MAP4K4, primary DRG neurons were electroporated with siRNAs directed against Tnik and Mink1. Both siRNAs resulted in effective knock-down, yet neither Tnik, Mink1 nor a combination of both Tnik and Mink1 blocked the induction of DLK or p-c-Jun (Fig. 4B). We therefore examined the possibility that all three GCK-IV subfamily kinases coordinately to regulate JNK pathway activation. To do this, knock-down of both Tnik and Mink1 was induced by electroporation of siRNA in Map4k4 deficient DRGs (Map4k4 $\left.4^{\text {loxp/loxp}} ; C_{r}{ }^{+}\right)$. Consistent with our MAP4K4 inhibitor treatment results, knock-down of Tnik and Mink1 in Map4k4-null neurons reduced the induction of p-JNK and p-c-Jun significantly after NGF withdrawal (Fig. 4C,D).

We next sought to determine whether the suppression of JNK signaling induced by loss of MAP4K4, MINK1, and TNIK expression was sufficient to elicit neuroprotection. This was done by treating Map4k4-deficient (Map4k $\left.4^{\text {loxp/loxp }} ; \mathrm{Cr}^{+}\right)$and control (Map4k $4^{\text {loxp/loxp }} ; \mathrm{Cr}^{-}$) DRGs with different combinations of siRNAs targeting Tnik and/or Mink1 (Fig. 4E). Consistent with our biochemical analysis (Fig. 4C,D), knock-down of all three kinases was required to achieve prominent rescue of axonal de- 
A

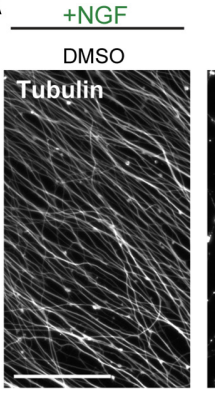

\section{DMSO}

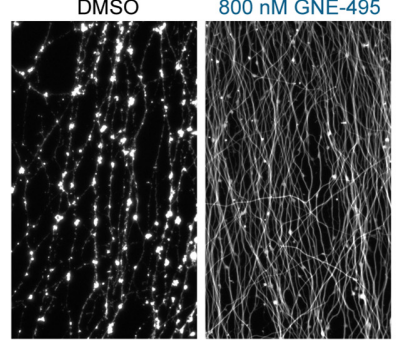

-NGF

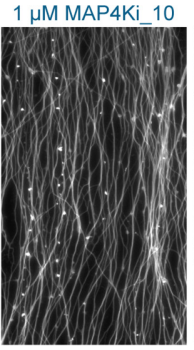

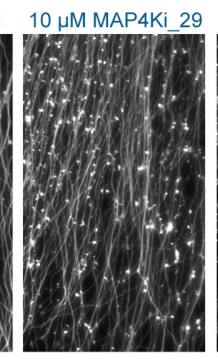

B

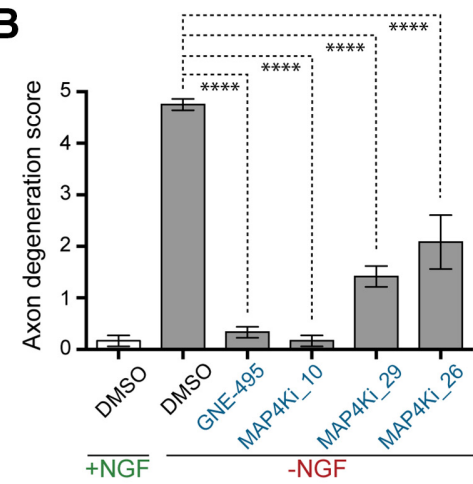

D

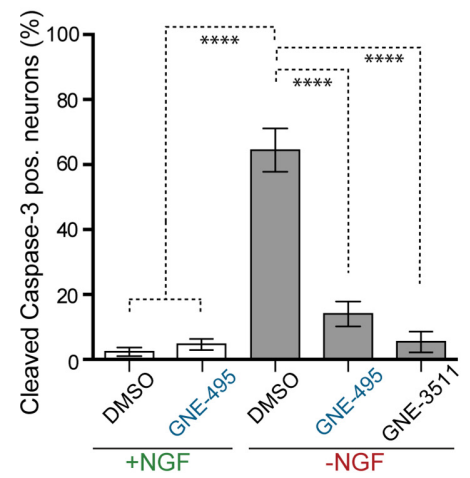

Figure 3. Inhibitors targeting MAP4K4 protects from axon degeneration and apoptosis after NGF withdrawal. $\boldsymbol{A}$, Four different inhibitors targeting MAP4K4 were able to block axon ( $\beta$-tubulin) degeneration in DRG explants $20 \mathrm{~h}$ after NGF withdrawal. $\boldsymbol{B}$, Quantification of axon degeneration in $\boldsymbol{A}$ using a five-point blinded scoring system (see Materials and Methods), demonstrating significant protection from axon fragmentation with inhibitors targeting MAP4K4: GNE-495, MAP4Ki_10,MAP4Ki_29, and MAP4Ki_26. $n=6$ /condition from 4 -6 explants. C, Treatment with GNE-495 blocks caspase-3 (red) activation in dissociated primary DRG neurons labeled with tubulin (green) $8 \mathrm{~h}$ after NGF withdrawal to a similar degree as DLK inhibition via treatment with GNE-3511. $n=6 /$ condition obtained from $4-6$ explants. D, Quantification of activated caspase- 3 in C calculated as percentage of caspase- 3 positive, $\beta$-tubulin-colabeled, neurons. $n=4-8$ wells per condition from 2 independent experiments (average of 2 fields of view per well, $20 \times$ ). Scale bars, $100 \mu m$. One-way ANOVA followed by Bonferroni post hoc test was used for statistical comparison. Data are represented as mean \pm SEM, ${ }^{* * * *} p<0.0001$.

generation (Fig. 4E,F). A partial rescue of axon degeneration was observed combining Map4k4 deletion and Tnik knock-down (Fig. 4E,F); however, no other combination resulted in significant axonal protection. Based on these findings, we conclude that these three kinases function redundantly to activate neuronal JNK signaling after NGF withdrawal, although MAP4K4 and TNIK may direct the majority of this effect.

After neuronal injury, DLK activation induces JIP3dependent retrograde propagation of stress signals, including p-JNK, from the axon to the nucleus (Ghosh et al., 2011; Shin et al., 2012; Huntwork-Rodriguez et al., 2013). We therefore sought to determine whether MAP4K4, MINK1, and TNIK act locally within axons to initiate retrograde DLK/JNK signaling. To do this, DRG explants were cultured on a porous membrane where distal axons could be readily separated from cell bodies/proximal axons (Unsain et al., 2014) (Fig. 5A, top schematic). Both DRG explant and axon samples were probed for MAP4K4, MINK1, and TNIK, all of which were readily detectable in DRG axons (Fig. 5A). NGF withdrawal did not alter the levels of these kinases substantially in either explants or isolated axons. Blotting for the phosphorylated form of the transcription factor c-Jun ( $\mathrm{p}-\mathrm{c}-\mathrm{Jun}$ ), which is present only in the nucleus, was included as a control for the purity of the axonal preparation. As expected, the levels of p-c-Jun were increased substantially after NGF withdrawal in the cell body compartment only (Fig. 5A).

Using the same assay setup, we next investigated whether MAP4K4, MINK, and TNIK regulate the retrograde transloca- tion of p-JNK to the nucleus that occurs after NGF deprivation. Withdrawal of NGF led to increased p-JNK in the cell body compartment beginning at $3 \mathrm{~h}$ (Fig. $5 B, D$ ), consistent with the time point at which robust $\mathrm{p}-\mathrm{c}-\mathrm{Jun}$ was visible in the same compartment (Fig. $5 B, C$ ). p-JNK levels in the axonal compartment were increased by $1 \mathrm{~h}$ of NGF deprivation, further increased at $3 \mathrm{~h}$, and declined at the $6 \mathrm{~h}$ time point (Fig. $5 B, D$ ). Treatment with GNE495 suppressed the p-JNK increase in the axonal compartment and prevented the appearance of $\mathrm{p}-\mathrm{JNK}$ in the cell body compartment, indicating that the retrograde translocation of $\mathrm{p}-\mathrm{JNK}$ was attenuated by compound treatment. This blockade of p-JNK signaling was sufficient to eliminate p-c-Jun induction in cell bodies (Fig. $5 B, C$ ). We then examined DLK protein abundance and molecular weight to determine how DLK phosphorylation correlates with p-JNK translocation. Total DLK levels in DRG axons decreased over time and this was unaffected by GNE-495 treatment (Fig. $5 E, F$ ). In contrast, total DLK levels in the cell body compartment increased in a time-dependent fashion after NGF withdrawal, which did not occur in neurons treated with GNE495 (Fig. 5E,F). Because the higher-molecular-weight form of DLK represents the active protein (Huntwork-Rodriguez et al., 2013), we also analyzed the proportion of activated DLK, as measured by the amount of shifted high-molecular-weight DLK normalized to total DLK (see illustration in Fig. 5). The active fraction of DLK increased dramatically in both axons and explants after NGF deprivation and was blocked efficiently by GNE-495 (Fig. 5G). 

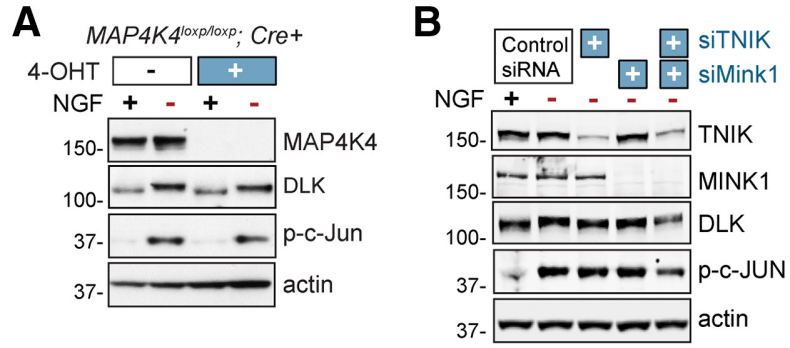

E
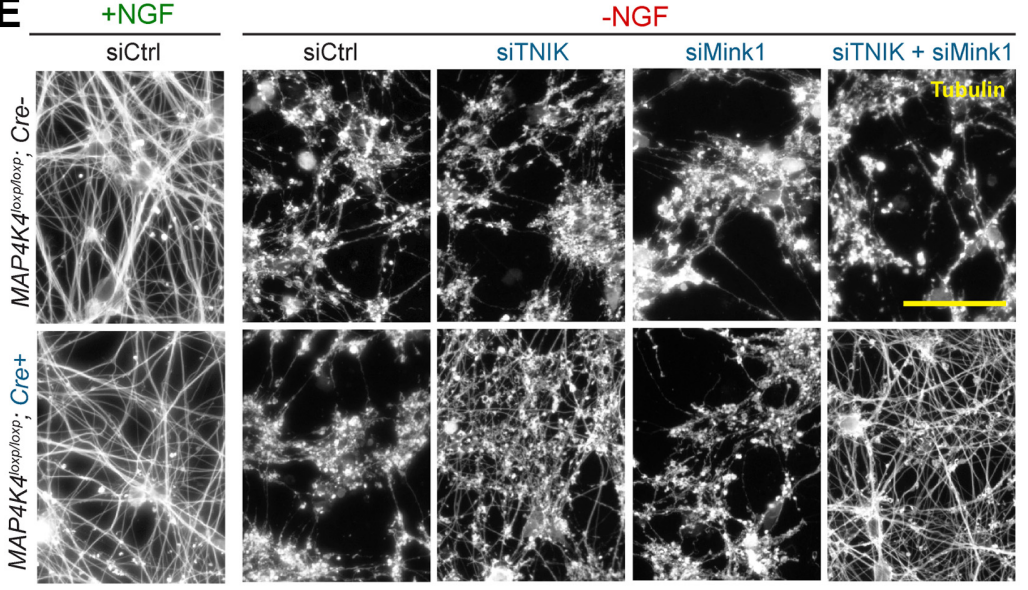
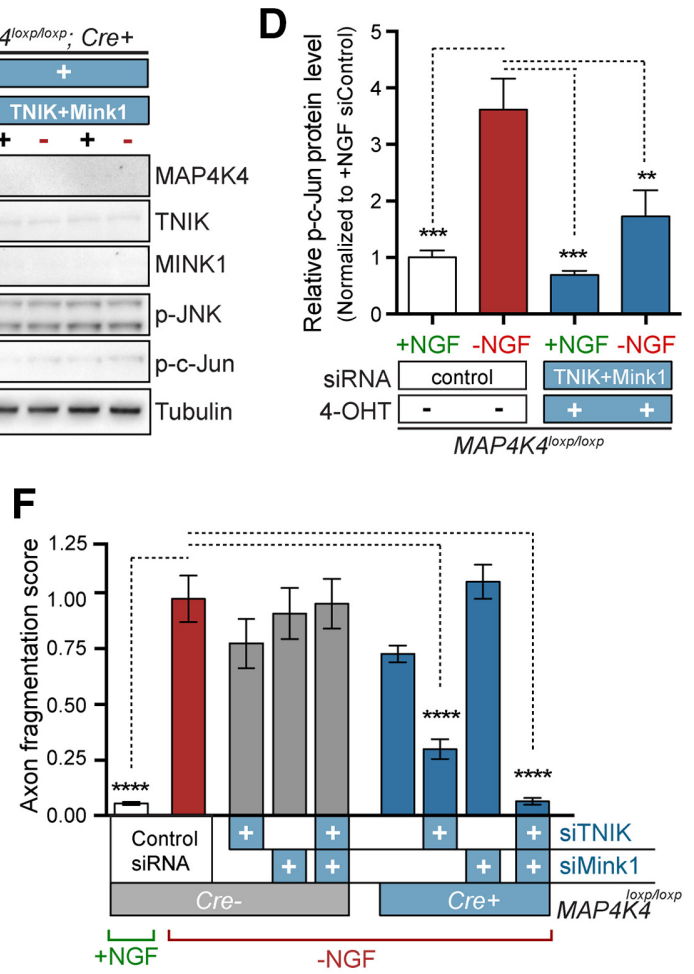

Figure 4. Knock-down of GCK-IV kinases MAP4K4, MINK1, and TNIK blocks JNK pathway driven neurodegeneration. $\boldsymbol{A}$, MAP4K4-deficient primary DRG neurons display normal activation of DLK and phosphorylation of c-Jun after NGF withdrawal. MAP4K4 ${ }^{\text {loxp/loxp }}$; Cre-ERT2 DRG neurons were treated with 4-0HT to delete Map4k4. B, siRNA-mediated knock-down of Tnik or Mink1 or both

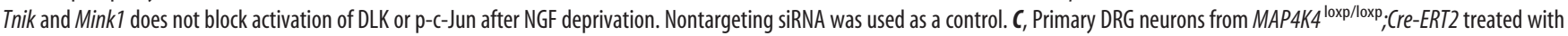
4-OHT and siRNAs for Tnik and Mink1 showed effective silencing of the three kinases. C, D, Triple knock-down of Map4k4, Mink1, and Tnik reduces JNK activation and p-C-Jun increase normally seen after NGF withdrawal after $2 \mathrm{~h}$ compared with Cre-DRGs electroporated with nontargeting control siRNA. Protein levels were normalized to $\beta$-tubulin and + NGF control siRNA samples (arbitrary units a.u.). $n=4-5$ /condition. $\boldsymbol{E}$, Representative immunofluorescence images of embryonic DRGs ( $\beta$-tubulin) after knock-down of all combinations of Map4k4, Mink1, and Tnik (including nontargeting siRNA controls) after $20 \mathrm{~h}$ of NGF withdrawal. Scale bar, $50 \mu \mathrm{m}$. $\boldsymbol{F}$, Loss of all three GCK-IV subfamily MAP4Ks results in neuroprotection. Knock-down of both Tnik and Map4k4 results in moderate neuroprotection. Axon fragmentation score was defined as the average number of connected axonal sets detected in each image divided by the mean number of connected axonal sets in the control -NGF condition of MAP4K4 ${ }^{\text {loxp/loxp }}$; $\left(r e{ }^{-}\right.$with control siRNA (which present degenerated axons). + NGF control: $n=32$ images; all other condition: $n=9$ images. The - NGF images were obtained from three different wells and the +NGF images were taken from one well of each condition. One-way ANOVA was used for statistical analysis comparing siRNA treatments with -NGF control, followed by a Dunnett's multiple-comparisons test. Data are represented as mean \pm SEM, ${ }^{* *} p<0.01,{ }^{* * *} p<0.001,{ }^{* * * *} p<0.0001$.

To confirm DLK translocation to the cell body after NGF deprivation, we next examined the localization of DLK and p-JNK in DRG neurons by immunofluorescence. Consistent with previous reports (Björkblom et al., 2008; Ghosh et al., 2011), we observed an increase in p-JNK intensity within cell bodies after $3 \mathrm{~h}$ of anti-NGF treatment (Fig. $5 H, I$ ). DLK staining was also increased in the cell bodies $3 \mathrm{~h}$ after NGF deprivation, whereas treatment with two different MAP4K4 inhibitors was sufficient to prevent the increase of both DLK and p-JNK in cell bodies upon NGF withdrawal (Fig. $5 \mathrm{H}, J$ ). Together, these results imply that MAP4K4, MINK, and TNIK regulate stress-induced retrograde translocation of DLK and p-JNK, with the majority of the DLK appearing in the cell body compartment being the active form of the kinase.

Interestingly, continual DLK signaling is required to maintain c-Jun phosphorylation and downstream transcriptional changes after optic nerve crush injury (Patel et al., 2015; Le Pichon et al., 2017). This can also be observed in DRG neurons, where addition of the DLK inhibitor GNE-3511 $3 \mathrm{~h}$ after NGF withdrawal completely reversed c-Jun phosphorylation at $4 \mathrm{~h}$, a result comparable to when the same compound was added at the time of NGF deprivation (Fig. $6 A-C$ ). This observation indicates that DLK kinase activity is required to maintain $\mathrm{p}$-c-Jun even after the retrograde JNK signal has reached the nucleus, further supporting the hypothesis that DLK is a component of this retrograde signal. We then investigated whether MAP4K4, MINK1, and TNIK are also required continually for JNK pathway activity or if they instead regulate activation DLK/JNK signaling in the axon. MAP4Ki_10 treatment was able to inhibit p-c-Jun induction potently when added at the initiation of NGF deprivation, but no reduction in $\mathrm{p}$-c-Jun was observed when the same compound was added after $3 \mathrm{~h}$ of NGF withdrawal (Fig. $6 \mathrm{~B}, C$ ). Together with the axonal localization of MAP4K4, MINK1, and TNIK (Fig. $5 A$ ), these data suggest that these MAP4Ks function to either initiate or propagate retrograde DLK/JNK signaling rather than to sustain DLK activation.

To further elucidate the mechanism by which these MAP4Ks regulate DLK/JNK signaling, we used compartmentalized Campenot chambers (Campenot, 1977) (Fig. 6D) in which distal DRG axons and proximal axons/cell bodies are separated into two microenvironments that can be treated with distinct pharmacological compounds. In this experimental setup, withdrawal of NGF from the distal axonal compartment is sufficient to trigger a retrograde stress response and results in phosphorylation of c-Jun even when NGF is present in the cell body compartment (Fig. $6 D, E$ ) (Ghosh et al., 2011). We reasoned that, if MAP4Ks act in the axon to regulate retrograde DLK/JNK signaling, then inhibition of MAP4Ks should block c-Jun phosphorylation when 
A
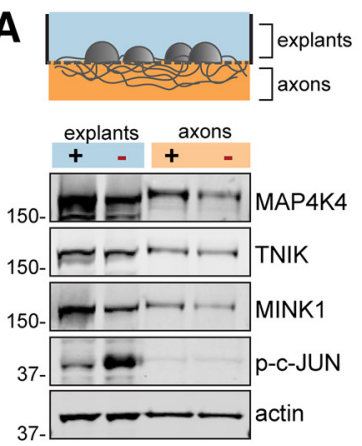

B

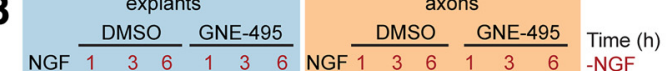

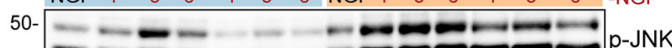

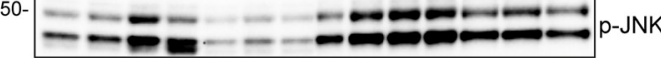

37-

$50-$

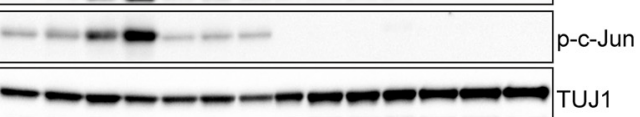

E

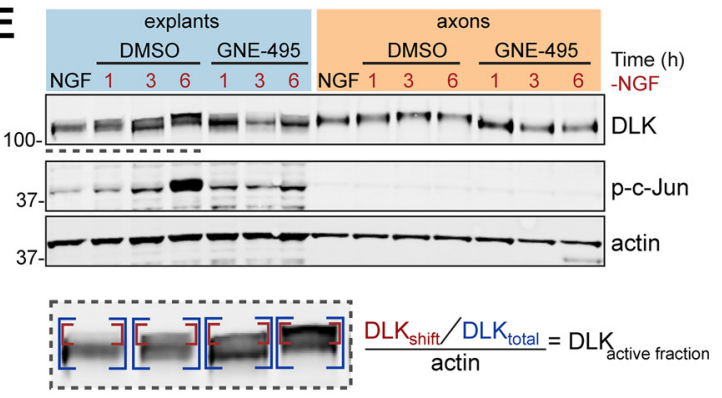

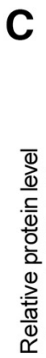

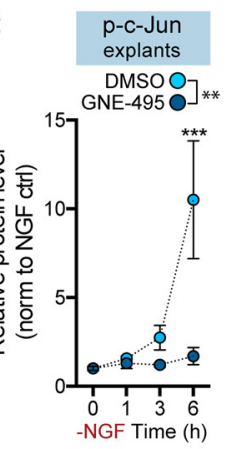

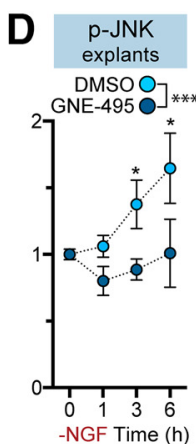

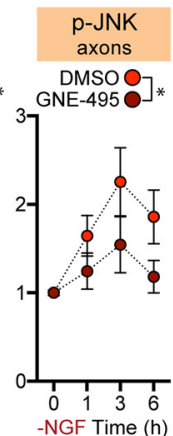

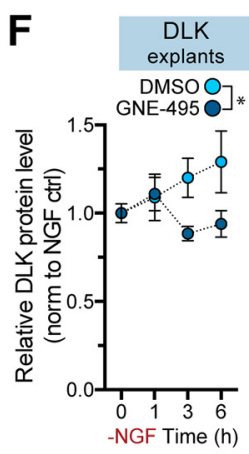
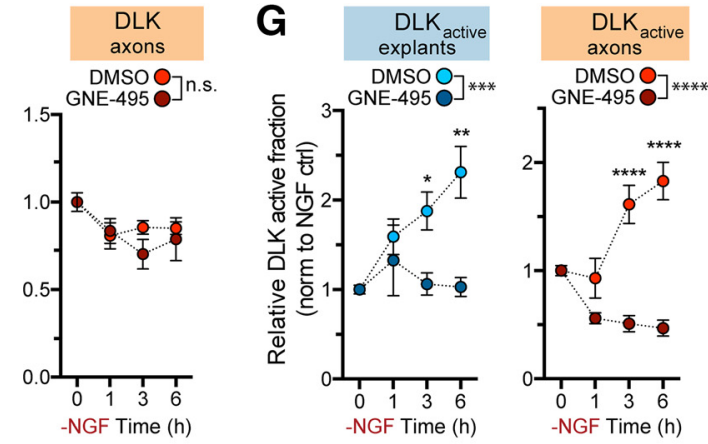

H
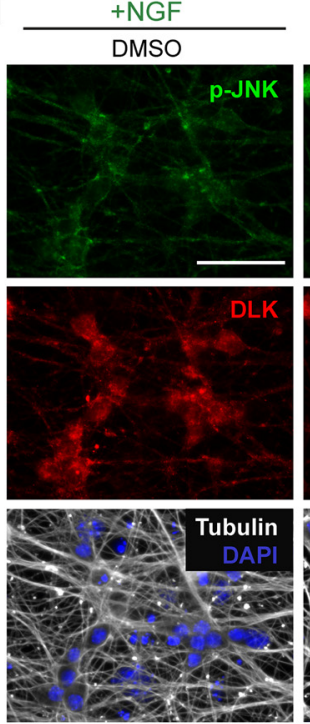
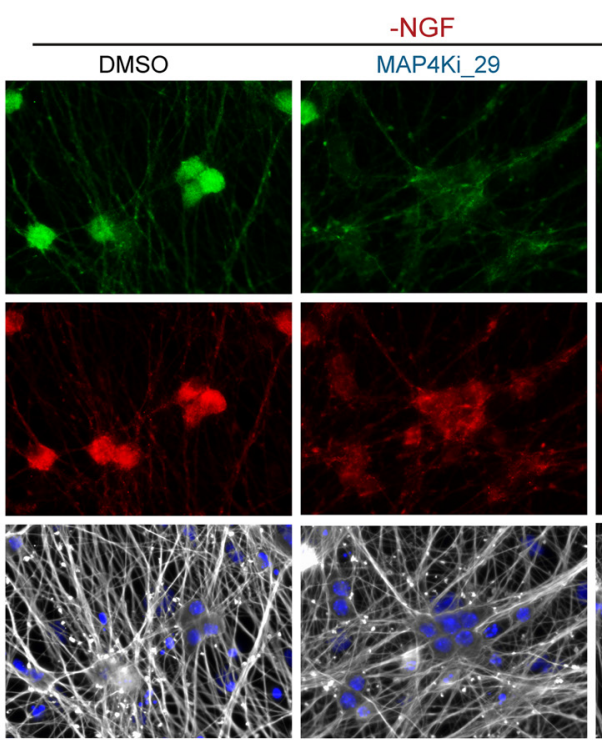
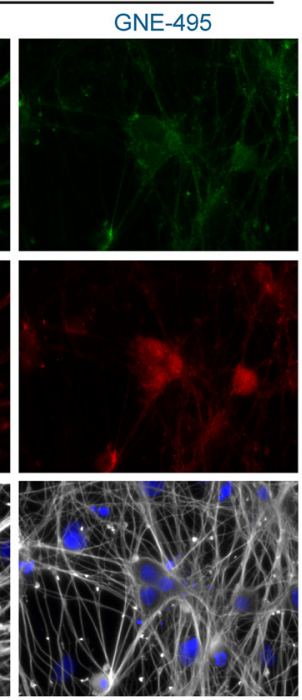
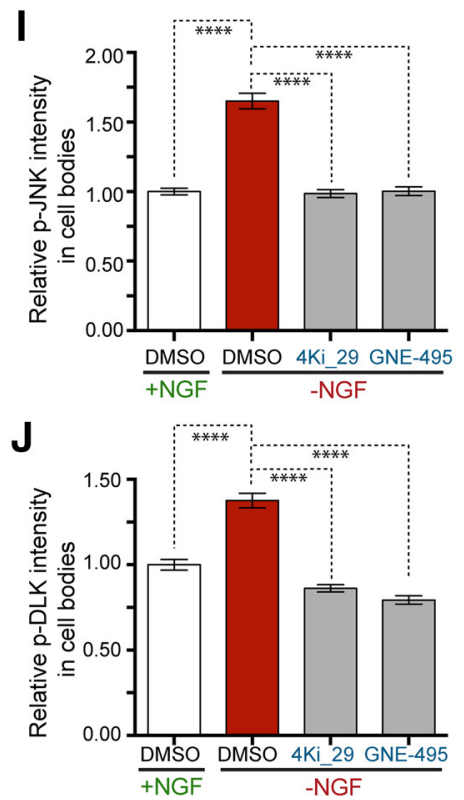

Figure 5. Inhibitors targeting MAP4K4, TNIK, and MINK1 block DLK/JNK pathway activation in axons and induction of DLK and p-JNK in the soma after NGF withdrawal. $A$, Schematic illustration of experimental setup for isolation of distal axons (lower compartment, orange) from DRG cell bodies/proximal axons (inner compartment, blue) of DRG explants. Western blot analysis of DRG cell bodies/proximal axons (left, blue) and axons (orange, right), demonstrates localization of MAP4K4, MINK1, and TNIK in isolated axons as well as cell bodies. $\boldsymbol{B}-\boldsymbol{G}$, Immunoblot analysis of isolated DRG axons and cell bodies after NGF-withdrawal at 1,3, and $6 \mathrm{~h}$ in the presence of DMSO or GNE-495. $\boldsymbol{B}-\boldsymbol{D}$, The presence of MAP4Ki GNE-495 reduced phosphorylation of $\mathrm{C}-\mathrm{Jun}$ in explants (blue) (C) and JNK in explants and axons (red) (D) after NGF deprivation compared with DMSO control. Immunoblot intensities were normalized to TUJ1 and + NGF control (0 h of NGF withdrawal). $p$-c-Jun: $n=4-5 /$ condition, $\mathrm{p}$-JNK: $n=8-9$ /condition. $\boldsymbol{E}$, Immunoblot analysis of DLK protein levels and $\mathrm{p}$-c-Jun in isolated DRG axons and cell bodies at 1,3 , and $6 \mathrm{~h}$ in the presence of DMSO or GNE-495 (top). Illustration at the bottom shows how the activated fraction of DLK was calculated based on the Western blot banding pattern: molecular-weight-shifted DLK shift $_{\text {(red) divided by total DLK }}$ total (blue) normalized to actin immunoblot band intensity. The dashed line highlights the magnified region of full blot. $F$, Total DLK protein levels increase in cell bodies/proximal axons over time, but

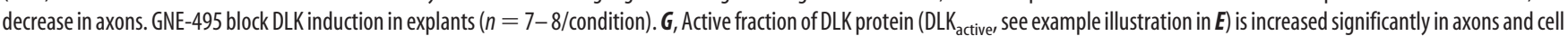
bodies/proximal axons over time. GNE-495 treatment prevents DLK activation in both axons and explants ( $n=7-8 /$ condition). $\boldsymbol{H}$, Immunofluorescence images demonstrating that both $p$-JNK (green) and DLK (red) protein is increased in the DRG cell bodies (DAPI: blue, TUJ-1: white) $3 \mathrm{~h}$ after NGF withdrawal. MAP4K inhibitors block the somal intensity of DLK and p-JNK. I, Quantification of somal p-JNK intensity relative to NGF p-JNK intensity in experiment H.J, Quantification of somal DLK intensity relative to NGF DLK intensity in experiment $\mathrm{H}$ ( $n=40$ cells/condition, obtained from 4 independent wells). One-way ANOVA followed by Bonferroni post hoc test was used for statistical comparison. Data are represented as mean \pm SEM. Statistical significance level between treatment for all time points are displayed above graphs in $\mathbf{C}, \mathbf{D}, \boldsymbol{F}$, and $\mathbf{G}$; statistical significance level of individual time points are presented in the graphs. ${ }^{*} p<0.05,{ }^{* *} p<0.01,{ }^{* * *} p<$ $0.001,{ }^{* * * *} p<0.0001$. 
A

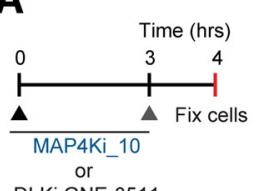

DLKi GNE-3511
B

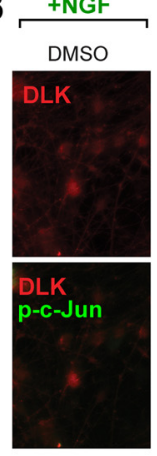

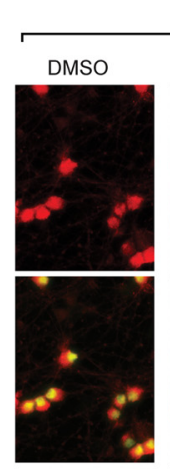

-NGF $(4$ hrs DLKi_GNE-3511
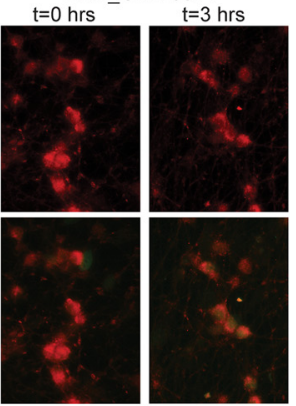

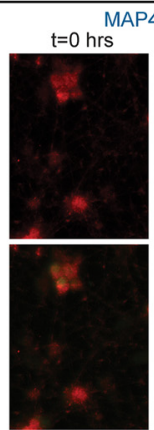

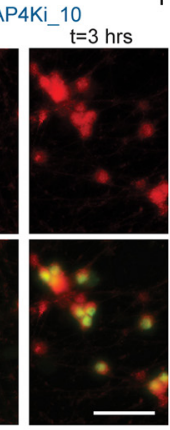

C

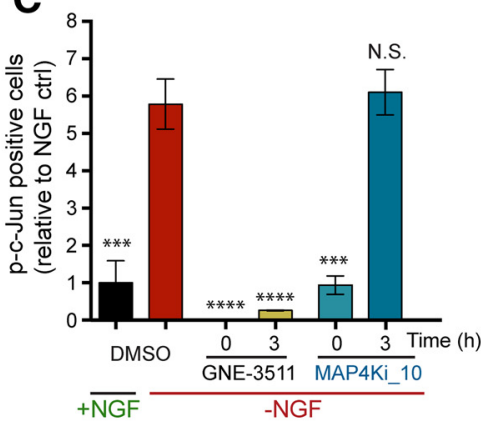

D

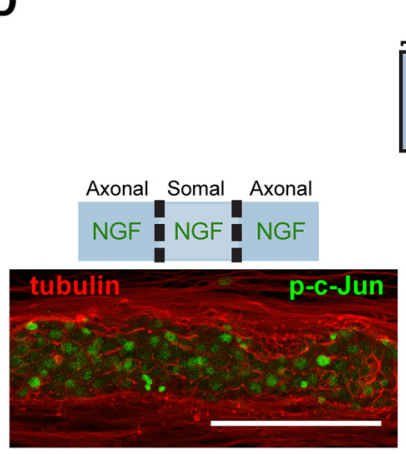

Campenot Chamber Axonal comp. Somal comp. Axonal comp.
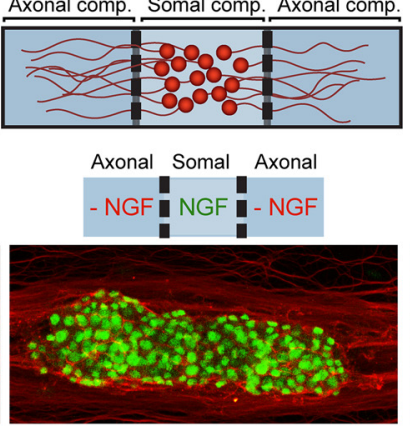

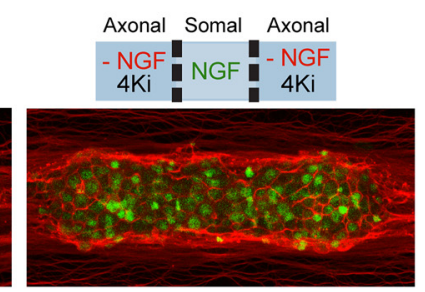

E

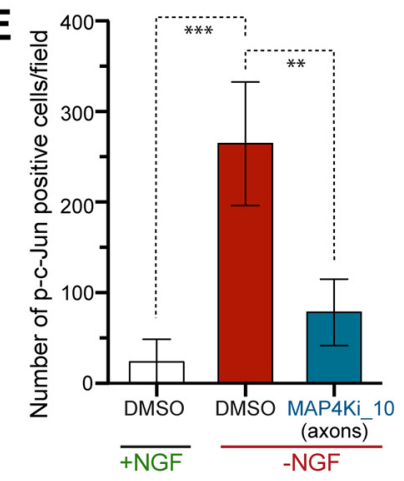

Figure 6. MAP4K4, MINK1, and TNIK are critical for retrograde DLK/JNK signaling after NGF deprivation. $\boldsymbol{A}$, Schematic of NGF withdrawal experiment performed in $\boldsymbol{B}$. MAP4K inhibitor (MAP4Ki_10, $10 \mu \mathrm{M}$ ) and DLK inhibitor (GNE-3511, $250 \mathrm{~nm}$ ) were added either at 0 or $3 \mathrm{~h}$ after NGF withdrawal from cultured embryonic DRGs. Cells were fixed after $4 \mathrm{~h}$. $\boldsymbol{B}$, MAP4K inhibition at the time of NGF withdrawal (MAP4Ki, $t=0$ ) reduces c-Jun phosphorylation; DLK inhibition (DLKi-3511) blocks c-Jun phosphorylation regardless of the timing of inhibitor addition. In contrast, inhibition of MAP4K after $3 \mathrm{~h}$ of NGF withdrawal (MAP4Ki, $t=3$ ) does not reduce p-c-Jun. Scale bar, $50 \mu \mathrm{m}$. C, Quantification of percentage $\mathrm{p}$-c-Jun-positive cells relative to + NGF control. For all conditions, $n=2$ images from 2 wells. D, Schematic illustration showing the compartmentalized Campenot chamber experimental layout (top). Dissociated DRGs (red) plated in the central (somal) compartment, is separated from the outer axonal compartment (see Materials and Methods for details). Diagrams of the experimental conditions are shown below: NGF control (left), NGF withdrawn from axons (middle) and NGF withdrawn from axons in the presence of MAP4K4 inhibitor (4Ki) (right). Representative images of somal compartments stained for p-c-Jun (green), as well as tubulin (red) to mark proximal axons are displayed for each condition. Scale bar, $100 \mu \mathrm{m}$. $\boldsymbol{D}, \boldsymbol{E}$, Addition of MAP4Ki_10 to the axonal compartment resulted in suppression of the p-c-Jun signal. $\boldsymbol{E}$, Quantification of the number of $\mathrm{p}$-c-Jun-positive nuclei ( $n=3-4 /$ condition). One-way ANOVA followed by Dunnett's post hoc test was used for statistical comparison. Data are represented as mean \pm SEM in $\boldsymbol{C}$ and mean \pm SD in $\boldsymbol{E}^{* *} p<0.01,{ }^{* * *} p<0.001,{ }^{* * * *} p<0.0001$. N.S., Nonsignificant.

added only to the distal axon compartment when NGF is removed. Indeed, addition of MAP4K inhibitor (MAP4Ki_10) to axons was sufficient to attenuate c-Jun phosphorylation (Fig. $6 D, E)$, thus supporting a model in which MAP4K4, MINK1, and TNIK regulate activation of retrograde DLK/JNK signaling in the axon.

\section{Discussion}

In this study, we identify the GCK-IV kinases MAP4K4, MINK1, and TNIK as regulators of DLK/JNK-signaling in neurons. This subfamily of Ste20 kinases has been shown previously to regulate a variety of cellular processes including angiogenesis, cellular polarization, and negative thymocyte selection (McCarty et al., 2005; Shitashige et al., 2010; Gloerich et al., 2012; Vitorino et al., 2015). Our findings are consistent with previous observations showing that overexpression of MAP4K4, MINK1, and TNIK can activate JNK in heterologous systems (Su et al., 1997; Fu et al., 1999; Hu et al., 2004; Machida et al., 2004). In the nervous system, TNIK and MINK1 have been identified as components of the postsynaptic density and regulate synaptic physiology (Hussain et al., 2010). MAP4K4 has been implicated in modulation of motor neuron degeneration based on data generated with Kenpaullone, a small molecule that inhibits both the MAP4Ks and GSK3 $\beta$ (Yang et al., 2013b). Using inhibitors specific for GCK-IV MAP4 kinases combined with loss-of-function studies, we demonstrate that MAP4K4 acts redundantly with the homologous kinases TNIK and MINK1 in the initiation of DLK/JNK signaling and translocation of activated JNK to the nucleus in neurons.

These findings provide an improved understanding of the factors that lead to DLK/JNK activation in the axon after neuronal injury, although it remains unclear how the GCK-IV MAP4 kinases themselves become activated. Some clues may come from the regulatory mechanisms of MAP4Ks identified in other systems. For instance, the Drosophila MAP4K homolog Misshapen has been shown to act downstream of kinase receptors (Liu et al., 1999; Paricio et al., 1999). In other settings, MAP4Ks appear to act downstream of small GTPases such as Rho or Rac (Manser et al., 1994; Brown et al., 1996; Dan et al., 2001). It is possible that MAP4K4, MINK1, and TNIK are activated via similar mechanisms after NGF deprivation; however, elucidation of the molecular pathways leading to MAP4K activation in mammalian neurons will require additional study.

How do MAP4K4, MINK1, and TNIK regulate the DLK/JNK pathway? Perhaps the most intuitive mechanism by which GCK-IV MAP4 kinases control JNK signaling would be through direct activation of MAP3 kinases such as DLK via phosphorylation of their activation loop. However, despite the nomenclature, a number of observations suggest that MAP4K-dependent modulation of DLK may not occur as part of a linear kinase cascade. First, DLK has been observed to undergo activation in the absence of any upstream kinase through dimerization-induced 
autophosphorylation. Indeed, dimerization of DLK protein is sufficient to result in phosphorylation of both the activation loop and other sites, such as T43, that regulate protein stability (Nihalani et al., 2000; Xiong et al., 2010; Huntwork-Rodriguez et al., 2013). Therefore, activation of DLK signaling may simply require induction of dimerization. Second, it was shown recently that another kinase, Akt, may represent a key signal that contributes directly to DLK activation. Akt can phosphorylate DLK in heterologous systems (Wu et al., 2015) and DLK-dependent neurodegeneration in DRGs can be initiated by a loss of Akt-mediated survival signaling (Simon et al., 2016). Third, there are few reports demonstrating that MAP4Ks are capable of phosphorylating a MAP3K in an endogenous cellular setting. Instead, MAP4Ks often drive cytoskeletal rearrangements or regulate cell motility in response to extracellular cues (Fu et al., 1999; Dan et al., 2001; Hu et al., 2004; Nonaka et al., 2008; Yue et al., 2014). Together, these observations suggest that GCK-IV MAP4Ks may facilitate propagation of DLK/JNK signaling to the nucleus indirectly rather than acting as an upstream kinase.

Recent findings have highlighted a central role for DLK in regulating the neuronal response to a variety of insults (Ghosh et al., 2011; Pozniak et al., 2013; Larhammar et al., 2017; Le Pichon et al., 2017), a function that is conserved in invertebrate species (Hammarlund et al., 2009; Yan et al., 2009; Xiong et al., 2010; Valakh et al., 2013). Given the pan-neuronal expression of MAP4K4, MINK1, and TNIK in the mouse brain, it is possible that MAP4Ks also regulate DLK signaling in these contexts. In Drosophila models, cAMP-mediated PKA activation has been shown to regulate DLK signaling (Hao et al., 2016), although this occurs through stabilization of DLK protein, thus other factors are also likely to contribute. The GCK-IV subfamily of Ste20 kinases is evolutionary conserved across multiple species, with orthologs in Drosophila and C. elegans (Dan et al., 2001), suggesting that this component of the signaling pathway may be similarly conserved. Further work will be needed to establish whether MAP4Ks or other distinct mechanisms regulate the DLK/JNK pathway in these settings.

\section{References}

Barnat M, Enslen H, Propst F, Davis RJ, Soares S, Nothias F (2010) Distinct roles of c-Jun N-terminal kinase isoforms in neurite initiation and elongation during axonal regeneration. J Neurosci 30:7804-7816. CrossRef Medline

Björkblom B, Vainio JC, Hongisto V, Herdegen T, Courtney MJ, Coffey ET (2008) All JNKs can kill, but nuclear localization is critical for neuronal death. J Biol Chem 283:19704-19713. CrossRef Medline

Brown JL, Stowers L, Baer M, Trejo J, Coughlin S, Chant J (1996) Human Ste20 homologue hPAK1 links GTPases to the JNK MAP kinase pathway. Curr Biol 6:598-605. CrossRef Medline

Campenot RB (1977) Local control of neurite development by nerve growth factor. Proc Natl Acad Sci U S A 74:4516-4519. CrossRef Medline

Cavalli V, Kujala P, Klumperman J, Goldstein LS (2005) Sunday Driver links axonal transport to damage signaling. J Cell Biol 168:775-787. CrossRef Medline

Coffey ET, Smiciene G, Hongisto V, Cao J, Brecht S, Herdegen T, Courtney MJ (2002) c-Jun N-terminal protein kinase (JNK) 2/3 is specifically activated by stress, mediating c-Jun activation, in the presence of constitutive JNK1 activity in cerebellar neurons. J Neurosci 22:4335-4345. Medline

Collins CA, Wairkar YP, Johnson SL, DiAntonio A (2006) Highwire restrains synaptic growth by attenuating a MAP kinase signal. Neuron 51: 57-69. CrossRef Medline

Crawford TD, et al. (2014) Discovery of selective 4-Amino-pyridopyrimidine inhibitors of MAP4K4 using fragment-based lead identification and optimization. J Med Chem 57:3484-3493. CrossRef Medline

Dan I, Watanabe NM, Kusumi A (2001) The Ste20 group kinases as regulators of MAP kinase cascades. Trends Cell Biol 11:220-230. CrossRef Medline
Fernandes KA, Harder JM, Fornarola LB, Freeman RS, Clark AF, Pang IH, John SW, Libby RT (2012) JNK2 and JNK3 are major regulators of axonal injury-induced retinal ganglion cell death. Neurobiol Dis 46:393401. CrossRef Medline

Fu CA, Shen M, Huang BC, Lasaga J, Payan DG, Luo Y (1999) TNIK, a novel member of the germinal center kinase family that activates the c-Jun $\mathrm{N}$-terminal kinase pathway and regulates the cytoskeleton. J Biol Chem 274:30729-30737. CrossRef Medline

Genabai NK, Ahmad S, Zhang Z, Jiang X, Gabaldon CA, Gangwani L (2015) Genetic inhibition of JNK3 ameliorates spinal muscular atrophy. Hum Mol Genet 24:6986-7004. CrossRef Medline

Ghosh AS, Wang B, Pozniak CD, Chen M, Watts RJ, Lewcock JW (2011) DLK induces developmental neuronal degeneration via selective regulation of proapoptotic JNK activity. J Cell Biol 194:751-764. CrossRef Medline

Gloerich M, ten Klooster JP, Vliem MJ, Koorman T, Zwartkruis FJ, Clevers H, Bos JL (2012) Rap2A links intestinal cell polarity to brush border formation. Nat Cell Biol 14:793-801. CrossRef Medline

Hammarlund M, Nix P, Hauth L, Jorgensen EM, Bastiani M (2009) Axon regeneration requires a conserved MAP kinase pathway. Science 323:802806. CrossRef Medline

Hao Y, Frey E, Yoon C, Wong H, Nestorovski D, Holzman LB, Giger RJ, DiAntonio A, Collins C (2016) An evolutionarily conserved mechanism for cAMP elicited axonal regeneration involves direct activation of the dual leucine zipper kinase DLK. Elife 5: pii: e14048. CrossRef Medline

Hirai S, Kawaguchi A, Hirasawa R, Baba M, Ohnishi T, Ohno S (2002) MAPK-upstream protein kinase (MUK) regulates the radial migration of immature neurons in telencephalon of mouse embryo. Development 129: 4483-4495. Medline

Hirai S, Kawaguchi A, Suenaga J, Ono M, Cui DF, Ohno S (2005) Expression of MUK/DLK/ZPK, an activator of the JNK pathway, in the nervous systems of the developing mouse embryo. Gene Expr Patterns 5:517-523. CrossRef Medline

Hu Y, Leo C, Yu S, Huang BC, Wang H, Shen M, Luo Y, Daniel-Issakani S, Payan DG, Xu X (2004) Identification and functional characterization of a novel human misshapen/Nck interacting kinase-related kinase, hMINK beta. J Biol Chem 279:54387-54397. CrossRef Medline

Hunot S, Vila M, Teismann P, Davis RJ, Hirsch EC, Przedborski S, Rakic P, Flavell RA (2004) JNK-mediated induction of cyclooxygenase 2 is required for neurodegeneration in a mouse model of Parkinson's disease. Proc Natl Acad Sci U S A 101:665-670. CrossRef Medline

Huntwork-Rodriguez S, Wang B, Watkins T, Ghosh AS, Pozniak CD, Bustos D, Newton K, Kirkpatrick DS, Lewcock JW (2013) JNK-mediated phosphorylation of DLK suppresses its ubiquitination to promote neuronal apoptosis. J Cell Biol 202:747-763. CrossRef Medline

Hussain NK, Hsin H, Huganir RL, Sheng M (2010) MINK and TNIK differentially act on Rap2-mediated signal transduction to regulate neuronal structure and AMPA receptor function. J Neurosci 30:14786-14794. CrossRef Medline

Larhammar M, Huntwork-Rodriguez S, Jiang Z, Solanoy H, Sengupta Ghosh A, Wang B, Kaminker JS, Huang K, Eastham-Anderson J, Siu M, Modrusan Z, Farley MM, Tessier-Lavigne M, Lewcock JW, Watkins TA (2017) Dual leucine zipper kinase-dependent PERK activation contributes to neuronal degeneration following insult. Elife 6: pii: e20725. CrossRef Medline

Le Pichon CE, et al. (2017) Loss of dual leucine zipper kinase signaling is protective in animal models of neurodegenerative disease. Sci Transl Med 9: pii: eaag0394. CrossRef Medline

Lindwall C, Kanje M (2005) Retrograde axonal transport of JNK signaling molecules influence injury induced nuclear changes in p-c-Jun and ATF3 in adult rat sensory neurons. Mol Cell Neurosci 29:269-282. CrossRef Medline

Liu H, Su YC, Becker E, Treisman J, Skolnik EY (1999) A Drosophila TNFreceptor-associated factor (TRAF) binds the ste20 kinase Misshapen and activates Jun kinase. Curr Biol 9:101-104. CrossRef Medline

Machida N, Umikawa M, Takei K, Sakima N, Myagmar BE, Taira K, Uezato H, Ogawa Y, Kariya K (2004) Mitogen-activated protein kinase kinase kinase kinase 4 as a putative effector of Rap2 to activate the c-Jun N-terminal kinase. J Biol Chem 279:15711-15714. CrossRef Medline

Manser E, Leung T, Salihuddin H, Zhao ZS, Lim L (1994) A brain serine/ threonine protein kinase activated by Cdc42 and Rac1. Nature 367:40 46. CrossRef Medline 
McCarty N, Paust S, Ikizawa K, Dan I, Li X, Cantor H (2005) Signaling by the kinase MINK is essential in the negative selection of autoreactive thymocytes. Nat Immunol 6:65-72. CrossRef Medline

Miller BR, Press C, Daniels RW, Sasaki Y, Milbrandt J, DiAntonio A (2009) A dual leucine kinase-dependent axon self-destruction program promotes Wallerian degeneration. Nat Neurosci 12:387-389. CrossRef Medline

Ndubaku C, et al. (2015) Structure-based design of GNE-495, a potent and selective MAP4K4 inhibitor with efficacy in retinal angiogenesis. ACS Med Chem Lett 6:913-918. CrossRef Medline

Nihalani D, Merritt S, Holzman LB (2000) Identification of structural and functional domains in mixed lineage kinase dual leucine zipper-bearing kinase required for complex formation and stress-activated protein kinase activation. J Biol Chem 275:7273-7279. CrossRef Medline

Nikolaev A, McLaughlin T, O'Leary DD, Tessier-Lavigne M (2009) APP binds DR6 to trigger axon pruning and neuron death via distinct caspases. Nature 457:981-989. CrossRef Medline

Nix P, Hisamoto N, Matsumoto K, Bastiani M (2011) Axon regeneration requires coordinate activation of p38 and JNK MAPK pathways. Proc Natl Acad Sci U S A 108:10738-10743. CrossRef Medline

Nonaka H, Takei K, Umikawa M, Oshiro M, Kuninaka K, Bayarjargal M, Asato T, Yamashiro Y, Uechi Y, Endo S, Suzuki T, Kariya KI (2008) MINK is a Rap2 effector for phosphorylation of the postsynaptic scaffold protein TANC1. Biochem Biophys Res Commun 377:573-578. CrossRef Medline

Oppenheim RW (1991) Cell death during development of the nervous system. Annu Rev Neurosci 14:453-501. CrossRef Medline

Paricio N, Feiguin F, Boutros M, Eaton S, Mlodzik M (1999) The Drosophila STE20-like kinase misshapen is required downstream of the Frizzled receptor in planar polarity signaling. EMBO J 18:4669-4678. CrossRef Medline

Patel S, et al. (2015) Discovery of dual leucine zipper kinase (DLK, MAP3K12) inhibitors with activity in neurodegeneration models. J Med Chem 58:401-418. CrossRef Medline

Pozniak CD, Sengupta Ghosh A, Gogineni A, Hanson JE, Lee SH, Larson JL, Solanoy H, Bustos D, Li H, Ngu H, Jubb AM, Ayalon G, Wu J, ScearceLevie K, Zhou Q, Weimer RM, Kirkpatrick DS, Lewcock JW (2013) Dual leucine zipper kinase is required for excitotoxicity-induced neuronal degeneration. J Exp Med 210:2553-2567. CrossRef Medline

Ries V, Silva RM, Oo TF, Cheng HC, Rzhetskaya M, Kholodilov N, Flavell RA, Kuan CY, Rakic P, Burke RE (2008) JNK2 and JNK3 combined are essential for apoptosis in dopamine neurons of the substantia nigra, but are not required for axon degeneration. J Neurochem 107:1578-1588. CrossRef Medline

Rudhard Y, Sengupta Ghosh A, Lippert B, Böcker A, Pedaran M, Kramer J, Ngu H, Foreman O, Liu Y, Lewcock JW (2015) Identification of 12/15lipoxygenase as a regulator of axon degeneration through high-content screening. J Neurosci 35:2927-2941. CrossRef Medline

Shin JE, Cho Y, Beirowski B, Milbrandt J, Cavalli V, DiAntonio A (2012) Dual leucine zipper kinase is required for retrograde injury signaling and axonal regeneration. Neuron 74:1015-1022. CrossRef Medline

Shitashige M, Satow R, Jigami T, Aoki K, Honda K, Shibata T, Ono M, Hirohashi S, Yamada T (2010) Traf2- and Nck-interacting kinase is essential for Wnt signaling and colorectal cancer growth. Cancer Res 70:50245033. CrossRef Medline
Simon DJ, Weimer RM, McLaughlin T, Kallop D, Stanger K, Yang J, O’Leary DD, Hannoush RN, Tessier-Lavigne M (2012) A caspase cascade regulating developmental axon degeneration. J Neurosci 32:17540-17553. CrossRef Medline

Simon DJ, Pitts J, Hertz NT, Yang J, Yamagishi Y, Olsen O, Tešić Mark M, Molina H, Tessier-Lavigne M (2016) Axon degeneration gated by retrograde activation of somatic pro-apoptotic signaling. Cell 164:1031-1045. CrossRef Medline

Su YC, Han J, Xu S, Cobb M, Skolnik EY (1997) NIK is a new Ste20-related kinase that binds NCK and MEKK1 and activates the SAPK/JNK cascade via a conserved regulatory domain. EMBO J 16:1279-1290. CrossRef Medline

Unsain N, Heard KN, Higgins JM, Barker PA (2014) Production and isolation of axons from sensory neurons for biochemical analysis using porous filters. J Vis Exp 89. CrossRef Medline

Valakh V, Walker LJ, Skeath JB, DiAntonio A (2013) Loss of the spectraplakin short stop activates the DLK injury response pathway in Drosophila. J Neurosci 33:17863-17873. CrossRef Medline

Vitorino P, Yeung S, Crow A, Bakke J, Smyczek T, West K, McNamara E, Eastham-Anderson J, Gould S, Harris SF, Ndubaku C, Ye W (2015) MAP4K4 regulates integrin-FERM binding to control endothelial cell motility. Nature 519:425-430. CrossRef Medline

Watkins TA, Wang B, Huntwork-Rodriguez S, Yang J, Jiang Z, EasthamAnderson J, Modrusan Z, Kaminker JS, Tessier-Lavigne M, Lewcock JW (2013) DLK initiates a transcriptional program that couples apoptotic and regenerative responses to axonal injury. Proc Natl Acad Sci U S A 110:4039-4044. CrossRef Medline

Wu CC, Wu HJ, Wang CH, Lin CH, Hsu SC, Chen YR, Hsiao M, Schuyler SC, Lu FL, Ma N, Lu J (2015) Akt suppresses DLK for maintaining selfrenewal of mouse embryonic stem cells. Cell Cycle 14:1207-1217. CrossRef Medline

Xiong X, Wang X, Ewanek R, Bhat P, Diantonio A, Collins CA (2010) Protein turnover of the Wallenda/DLK kinase regulates a retrograde response to axonal injury. J Cell Biol 191:211-223. CrossRef Medline

Xue Y, Wang X, Li Z, Gotoh N, Chapman D, Skolnik EY (2001) Mesodermal patterning defect in mice lacking the Ste20 NCK interacting kinase (NIK). Development 128:1559-1572. Medline

Yan D, Wu Z, Chisholm AD, Jin Y (2009) The DLK-1 kinase promotes mRNA stability and local translation in C. elegans synapses and axon regeneration. Cell 138:1005-1018. CrossRef Medline

Yang J, Weimer RM, Kallop D, Olsen O, Wu Z, Renier N, Uryu K, TessierLavigne M (2013a) Regulation of axon degeneration after injury and in development by the endogenous calpain inhibitor calpastatin. Neuron 80:1175-1189. CrossRef Medline

Yang YM, Gupta SK, Kim KJ, Powers BE, Cerqueira A, Wainger BJ, Ngo HD, Rosowski KA, Schein PA, Ackeifi CA, Arvanites AC, Davidow LS, Woolf CJ, Rubin LL (2013b) A small molecule screen in stem-cell-derived motor neurons identifies a kinase inhibitor as a candidate therapeutic for ALS. Cell Stem Cell 12:713-726. CrossRef Medline

Yue J, Xie M, Gou X, Lee P, Schneider MD, Wu X (2014) Microtubules regulate focal adhesion dynamics through MAP4K4. Dev Cell 31:572585. CrossRef Medline 